\title{
Acute Massive Pulmonary Embolism (AMPE): From Therapeutic to Protective Management - A Case Report
}

\author{
AQM Reza ${ }^{1}$, Attawar Sandeep $\mathrm{G}^{2}$, S Munwar ${ }^{1}$, AB Siddique ${ }^{3}$, SU Talukder ${ }^{1}$, \\ A Hasan ${ }^{4}$, AHMW Islam ${ }^{1}$ \\ ${ }^{1}$ Department of Clinical \& Interventional Cardiology, Apollo Hospitals, Dhaka, ${ }^{2}$ Department of \\ Cardiovascular \& Thoracic Surgery, Apollo Hospitals, Dhaka, ${ }^{3}$ Department of Cardiology, Apollo \\ Hospitals, Dhaka, ${ }^{4}$ Department of Internal Medicine, Apollo Hospitals, Dhaka
}

Key words: Massive, Pulmonary embolism,

(Cardiovasc. j. 2011; 3(2): 213-217) Embolectomy, IVC Filter.

\section{Introduction:}

Massive Pulmonary Embolism (PE) is life threatening. ${ }^{1} \mathrm{PE}$ should be suspected in all patients who presents with new or worsening dyspnoea, chest pain or sustained hypotension without an alternative obvious cause. ${ }^{2}$ Massive $\mathrm{PE}$ - that which is associated with more than $50 \%$ occlusion of the pulmonary arteries - may be associated with systemic hypotension, pulmonary hypertension and an increased mortality rate. Diagnosis is usually established with computed tomography scanning, pulmonary angiography, ventilation-perfusion scans, or echocardiography. Therapy generally consists of anticoagulation, thrombolysis, and rarely pulmonary embolectomy. ${ }^{3}$ We describe the case of a patient with acute massive pulmonary embolism, from Deep Venous Thrombosis of right popliteal vein in the setting of significant paraparesis due to previous history of GuillainBarre' Syndrome (GBS), which was managed successfully in our centre.

\section{Case Report:}

A 42 years old normotensive, non-diabetic, lean body built Bangladeshi lady got admitted on 24.05.2010 afternoon in Apollo Hospitals Dhaka with the complaints of chest compression, shortness of breath with profuse sweating for 1 day. She had similar type of episode 1 day back in aircraft while traveling from abroad. In emergency her pulse was 120/min regular and BP was 80/40 mmHg. ECG showed sinus tachycardia without any significant ST\&T changes. Immediately she was treated with high flow O2, I/V ionotrope and fluid to maintain her $\mathrm{BP}$ and continuous monitoring of vitals including central venous pressure monitoring. Her cardiac markers were inconclusive; S. Troponin-I $0.22 \mathrm{ng} / \mathrm{ml}$. X-ray chest showed no significant abnormality. Serial ECG showed gradual appearance of S1Q3T3 changes, suggestive of massive Pulmonary Embolism. Bedside echo showed dilated RA and RV with RV free wall hypokinesia and systolic dysfunction with moderately high Pulmonary systolic pressure (60 $\mathrm{mmHg})$. LV was normal in size with good regional and global wall motion. D-dimer was $821 \mathrm{mic} \mathrm{gm} /$ lit. Immediately CT Pulmonary Angiogram was done. It revealed a saddle thrombus in the bifurcation of main pulmonary artery extending into the both branches (Fig. 1, 2). Bilateral segmental and subsegmental branches involved having acute thrombus within causing partial obliteration of lumen with no definite cut off. Right sided pleural effusion with early basal atelectatic changes. Lower limbs Doppler showed Acute to early subacute thrombosis in right Popliteal vein. She had a significant past history of Guillain- Barre' Syndrome (GBS) with significant paraparesis of lower limbs for which she was admitted in same hospital 2 months back and discharged home with advice of regular physiotherapy of lower limbs. During this admission she also had to take assist from other during walking. Her treatment started conservatively with IV heparin \& fluids. I/V thrombolysis was deferred due to high risk of complications like intracerebral haemorrage. 


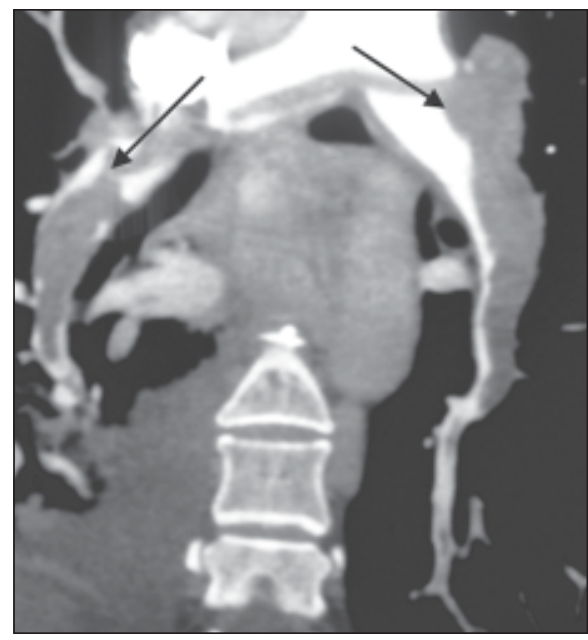

Fig.-1: Saddle thrombus in MPA

The following day as her BP was not raising with adequate inotropes, pulse rate was $>130 / \mathrm{min}$ and low volume and her $\mathrm{O} 2$ saturation was not maintaining with out high flow through mask and her chest discomfort and short of breath persisted, she was advised for Surgical thrombectomy and was immediately transferred to department of CT Surgery on same day. Clots were removed surgically under cardiopulmonary bypass (Fig. 3, 4). By Fogerty emboletomy rest of the clots removed from distal branches. SVC transected to gain access to RPA. RPA opened by longitudinal incision. More clots found here and removed directly and by Fogerty embolectomy. Both the PAs flushed by heparin saline. RPA and LPA closed directly. Her postoperative period was uneventful. From $2^{\text {nd }}$ POD LMWH twice daily with Aspirin/ clopidogrel and warfarin were started. On $4^{\text {th }}$ POD she was shifted to cabin. Then we planned to

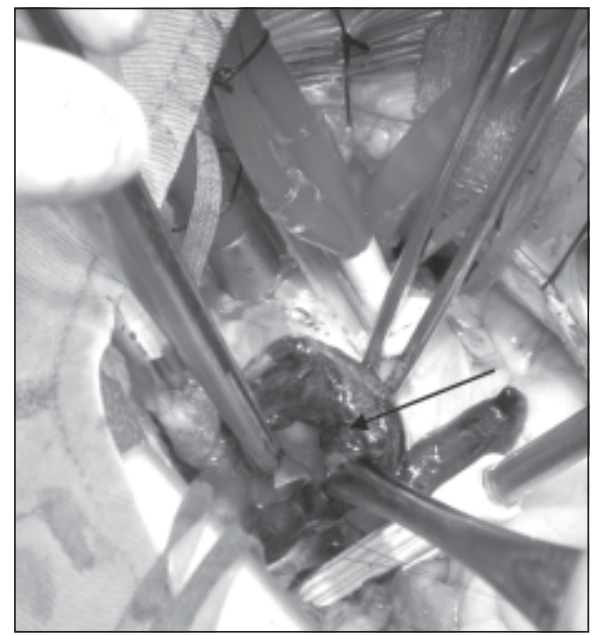

Fig.-3: Thrombus extraction from $M P A$

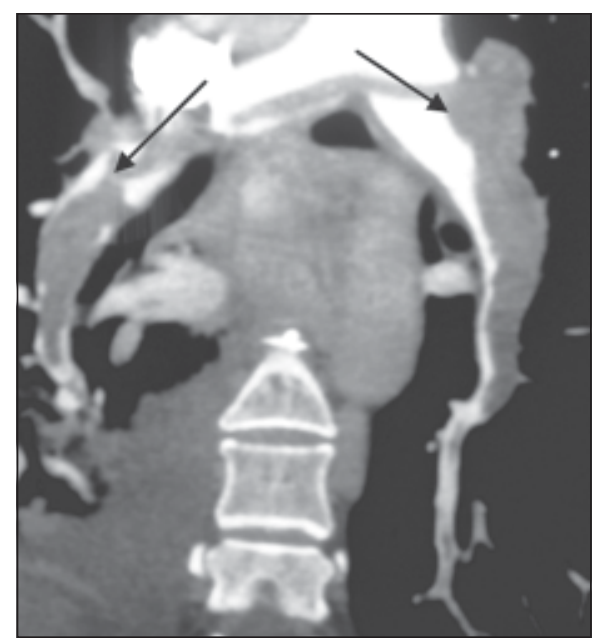

Fig.-2: Huge thrombus in RPA \& LPA

implant an IVC filter to protect further recurrence of PE.

On $6^{\text {th }}$ POD patient was transferred to Cath Lab for IVC Filter implantation. With all aseptic precautions, as there was subacute thrombosis in right Popliteal vein we approached through left Femoral vein. IVC venogram was done. A 6 F JR 3.5 catheter was used for selective canulation of renal veins. The position of the Filter was carefully selected. Then with the introducer Bard Filter was deployed $1 \mathrm{~cm}$ below the right Renal vein (which is the lower one) in the IVC. After implantation again IVC Venogram was done to see position \& alignment (Fig. 5, 6), which revealed excellent result. Then she was closely observed in CCU for 2 hours and then shifted to Cabin. Patient was discharged on $8^{\text {th }}$ June and advised to come for follow up at 7 days, 30 days \& 90 days. Patient was reviewed accordingly \& she was doing fine.

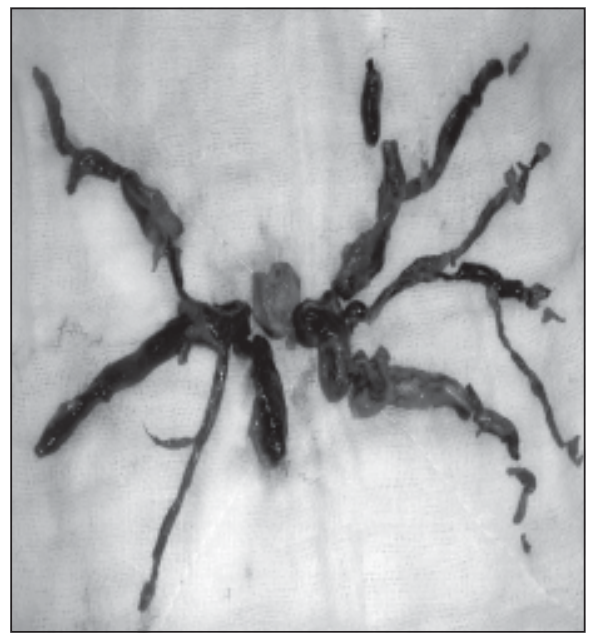

Fig.-4: Huge extracted thrombus arranged as located within PA\& its branches. 


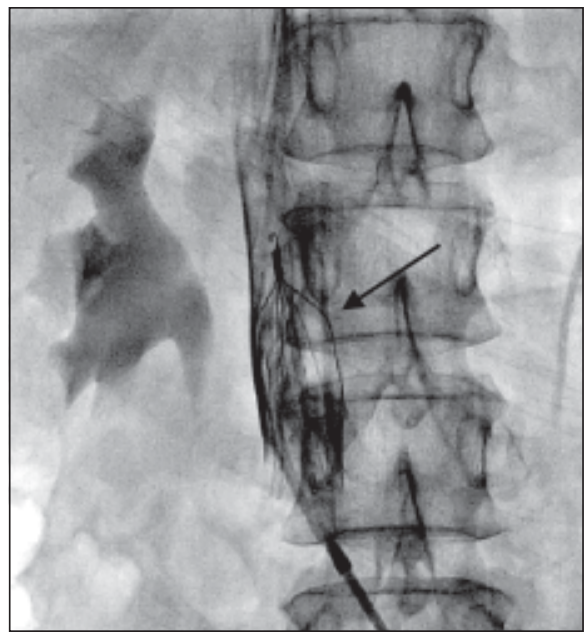

Fig.-5: Venogram during Filter implant.

\section{Discussion:}

Venous thromboembolism (VTE) has an incidence of approximately 100 individuals per 100,000 population each year in the United States. ${ }^{4}$ The most common presentation of VTE is deep venous thrombosis (DVT) or its more feared complication, pulmonary embolism (PE). Each year VTE is responsible for the deaths of at least 100,000 to 200,000 patients in the United States who have an otherwise good prognosis and whose deaths could likewise be preventable. ${ }^{5}$

First described in the 1800s, pulmonary embolism can be classified as acute or chronic, as submassive $(25 \%-50 \%$ obstruction) or massive $(>50 \%$ obstruction), and as central or peripheral. $\underline{6}$ Acute pulmonary embolism leads to an abrupt rise in pulmonary vascular resistance. Right ventricular contractile function is compromised, and right ventricular failure ensues. This vicious cycle of cardiogenic shock is augmented by concomitant hypoxia, which inevitably leads to cardiovascular collapse. $\underline{T}$ The interval from the onset of symptoms to death is relatively short. In patients with massive pulmonary embolism, $50 \%$ die within 30 minutes, $70 \%$ die within 1 hour, and more than $85 \%$ die within 6 hours of the onset of symptoms.

The principals of treatment of massive PE includes reducing the clot burden by thrombolysis or mechanical removal and prevention of further clot production (DVT by anticoagulation - heparin and warfarin) and clot migration by caval filter insertion. $\underline{9}$

Treatment options for massive pulmonary embolism vary, depending on the clinical picture

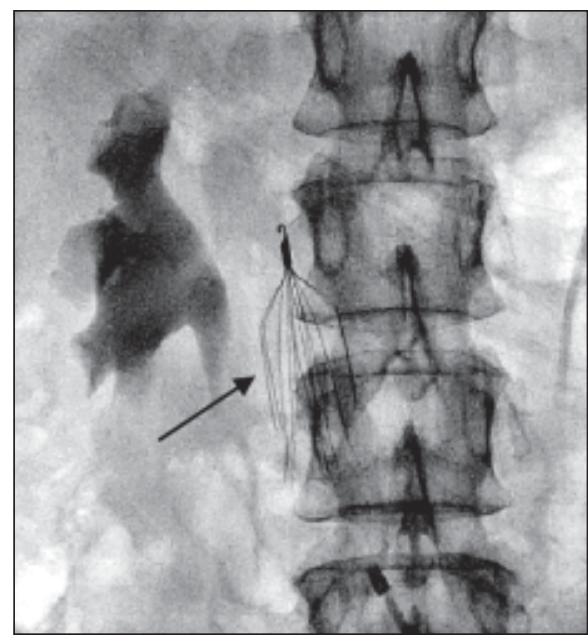

Fig.-6. IVC filter in situ after implant.

of the patient. Although anticoagulation and thrombolysis are the standard for treatment of acute massive pulmonary embolism, these treatments are limited to patients who are hemodynamically stable and do not have contraindications. Furthermore, data suggest that patients treated with thrombolysis have a higher death rate, increased risk of major hemorrhage, and increased rates of recurrence of pulmonary embolism, compared with patients treated by means of pulmonary embolectomy. 10 The International Cooperative Pulmonary Embolism Registry found a surprisingly high intracranial hemorrhage rate of $3 \%$ among patients with pulmonary embolism who were treated with thrombolytic therapy. ${ }^{11}$ However, these procedures are associated with an increased risk of hemorrhage, recurrent emboli, and failure to completely resolve the intraluminal thrombus, putting patients at higher risk to develop pulmonary hypertension. ${ }^{12,13,14}$

Early surgical treatment must also be considered in patients whose course deteriorates in spite of aggressive medical therapy. $\frac{15}{15}$ Depending on the series, the overall mortality rate after pulmonary embolectomy varies from $16 \%$ to $46 \%$, with a mean mortality rate of 26\%. $\underline{6}$ Ullmann's group reported an operative mortality of $35 \%$ in their series. ${ }^{6}$ The high mortality rate, for the most part, is due to the fact that most patients who undergo surgical embolectomy are hemodynamically compromised and arrive at the operating room in cardiac arrest with cardiopulmonary resuscitation $(\mathrm{CPR})$ in 
progress, or else they have had CPR performed beforehand. Data suggest that preoperative hemodynamic status is the most important prognostic indicator of postoperative outcome after surgical pulmonary embolectomy, and cardiac arrest and CPR are independent factors predictive of postoperative death. $\frac{6,15}{}$ These findings suggest that earlier surgical intervention may result in improved survival. The present report describes a case in which severe hemodynamic compromise mandated a surgical embolectomy.

Patients with acute PE are at risk for recurrent thromboembolic events, mainly a second PE. The risk of recurrent $\mathrm{PE}$ is less than $1 \%$ per year while patients are receiving anticoagulant therapy, but the risk is 2 to $10 \%$ per year after the discontinuation of such therapy. $\frac{16}{2}$ Risk factors for recurrence include male sex, advanced age and idiopathic or unprovoked PE can be as high as 50\% among patients with $\mathrm{PE}$. The risk of recurrence is particularly high among patients with cancer. The risk of recurrence is about 3\% per year among patients in whom the first $\mathrm{PE}$ was associated with a temporary risk factor, such as major surgery, immobilization because of an acute medical illness, or trauma. ${ }^{2}$

After an acute PE, patients should be monitored for chronic thromboembolic pulmonary hypertension. The incidence of chronic thromboembolic pulmonary hypertension 2 years after the acute event ranges from 0.8 to $3.8 \% .{ }^{17,18}$

Vena cava filters were introduced in the 1960s as a mechanical means to prevent PE. ${ }^{19}$ Since that time, the number of filters placed has grown steadily to over 40,000 annually in the United States alone. ${ }^{20}$ Randomized trials have shown that in addition to heparin therapy, the use of permanent filters initially reduces the occurrence of symptomatic or asymptomatic $\mathrm{PE}$ without changes in either immediate or long-term mortality. ${ }^{21}$

In this case we decided to implant vena caval filter to prevent further devastating recurrence of PE.

\section{Conclusion:}

Massive pulmonary embolism remains a highly fatal disease. An episode of severe hypotension in a patient with deep vein thrombosis and suspected pulmonary embolism requires rapid diagnosis to enable expedient thrombolysis or surgery. As thrombolysis bears higher rates of death, major haemorrhage and recurrent embolism than open pulmonary embolectomy, early referral to a cardiothoracic surgeon should thus be considered in cases of significant embolism. To prevent recurrence early IVC filter implantation may be a good choice to complete the treatment of AMPE.

\section{References:}

1. Nils Kucher, Samuel Z. Goldhaber. Clinical update. Management of Massive Pulmionary Embolism. Circulation 2005 ;112:e28-e32.

2. Giancarlo Agnelli, Cecilia Becattina. Current concepts: Acute Pulmonary Embolism. N Engl J Med 2010; 363:266-74

3. Gregory R Brevetti, Benjamin O’Brien, Cynara L. Coomer, Timothy S. Hall, Lucy S. Brevetti, and David M. Jablons. Emergency Surgery for Massive Pulmonary Embolism on the basis of clinical diagnosis. Tex Heart Inst $J$ 2003;30:149-51

4. Tschoe M, Kim HS, Brotman DJ, Streiff MB. Retrievable vena cava filters: A clinical review. J Hosp Med 2009;4:441-448.

5. Buller HR, Agnelli G, Hull RD, et al. Antithrombotic therapy for venous thromboembolic disease. The Seventh American College of Chest Physicians on antithrombotic and thrombolytic therapy. Chest 2004;126:401S-428S.

6. Ullmann M, Hemmer W, Hannekum A. The urgent pulmonary embolectomy: mechanical resuscitation in the operating theatre determines the outcome. Thorac Cardiovasc Surg 1999;47:5-8.

7. Ohteki H, Norita H, Sakai M, Narita Y. Emergency pulmonary embolectomy with percutaneous cardiopulmonary bypass. Ann Thorac Surg 1997;63:1584-6.

8. Stulz P, Schlapfer R, Feer R, Habicht J, Gradel E. Decision making in the surgical treatment of massive pulmonary embolism. Eur J Cardiothorac Surg 1994;8:188-93.

9. Goldhaber SZ. Pulmonary embolism. Review. N Engl J Med 1998;339:93- 104

10. Gulba D, Schmid C, Borst HG, Lichtlen P, Dietz R, Luft FC. Medical compared with surgical treatment for massive pulmonary embolism. Lancet 1994;343:576-7.

11. Goldhaber SZ, Visani L, De Rosa M. Acute pulmonary embolism: clinical outcomes in the International Cooperative Pulmonary Embolism Registry (ICOPER). Lancet 1999; 353:1386-9.

12. Gulba DC, Schmid C, Borst HG, Lichtlen P, Dietz R, Luft FC. Medical compared with surgical treatment for massive pulmonary embolism Lancet 1994;343:576-577. 
13. Yalamanchili K, Fleisher AG, Lehrman SG, et al. Open pulmonary embolectomy for treatment of major pulmonary embolism. Ann Thorac Surg 2004;77:819823

14. Kanter DS, Mikkola KM, Patel SR, Parker JA, Goldhaber SZ. Thrombolytic therapy for pulmonary embolism: frequency of intracranial hemorrhage and associated risk factors. Chest 1997;111:1241-1245

15. Meyer G, Tamisier D, Sors H, Stern M, Vouhe P, Makowski S, et al. Pulmonary embolectomy: a 20-year experience at one center. Ann Thorac Surg 1991;51:232-6.

16. Agnelli G, Prandoni P, Becattini C, et al. Extended oral anticoagulant therapy after a first episode of pulmonary embolism. Ann Intern Med 2003;139:19-25.
17. Pengo V, Lensing AWA, Prins MH, et al. Incidence of chronic thromboembolic pulmonary hypertension after pulmonary embolism. N Engl J Med 2004;350:2257-64.

18. Becattini C, Agnelli G, Pesavento R, et al. Incidence of chronic thromboembolic pulmonary hypertension after a first episode of pulmonary embolism. Chest 2006:130:172-5.

19. Mobin-Uddin K, Smit PE, Martinez LO, et al. A vena caval filter for the prevention of pulmonary embolus. Surg Forum 1967;18.

20. Stein PD, Kayali F, Olson RE. Twenty-one-year trends in the use of inferior vena cava filters. Arch Intern Med 2004;164:1541-1545.

21. Decousus H, Leizorowich A, Parent F, et al. A clinical trial of vena caval filters in the prevention of pulmonary embolism in patients with proximal deep-vein thrombosis. N Engl J Med 1998;338:409-415. 